Conclusions ANA IIF alone rare and patients infrequently develop nephritis, leucopenia, lymphocytopenia, AITP, AIHA. In patients ANA IIF alone attribution of ACR/SLICC clinical criteria needs to be point of emphasis and unless biopsy proven, alternative explanation for proteinuria should be considered. Data argues inclusion criteria for clinical trials, rather than allowing ANA IIF alone or dsDNA, may need to require ANA IIF and at least one of the following (dsDNA, Sm, Ro, $\mathrm{La}$, aPL, or hypocomplementemia) to avoid enrolling patients that do not have SLE.

Funding Source(s): None

\section{INCREASED WORK LOSS DURING PREGNANCY IN WOMEN WITH SYSTEMIC LUPUS ERYTHEMATOSUS COMPARED TO MATCHED HEALTHY CONTROLS}

${ }^{1}$ Birgit S Blomjous, ${ }^{2}$ Marieke M ter Wee, ${ }^{1}$ Alexandre E Voskuyl, ${ }^{3}$ Johanna IP de Vries, ${ }^{1}$ Irene EM Bultink*. ${ }^{1}$ Department of Rheumatology, Amsterdam Rheumatology and immunology Center, Amsterdam UMC, location VUmc, Amsterdam, Netherlands; 'Department of Epidemiology and Biostatistics, Amsterdam UMC, location VUmc, Amsterdam, Netherlands; ${ }^{3}$ Department of Obstetrics and Gynecology, Amsterdam UMC, location VUmc, Amsterdam, Netherlands

10.1136/lupus-2019-Ism.65

Background Women with systemic lupus erythematosus (SLE) might be more vulnerable to reduce or stop working during pregnancy because of the increased risk of pregnancy complications compared to the general population. However, no data on work loss during pregnancy and return to work after maternity leave in patients with SLE are available. We aimed to investigate several work outcomes during and after pregnancy in women with SLE compared to matched pregnant controls.

Methods A case-control study on employment was performed in pregnant women with SLE and matched controls. Matching criteria were age, year of delivery, and number of living infants. Employment was defined as having 8 hours/week of paid work before conception. Four work outcomes were investigated: interruption of work for $>1$ week during pregnancy, complete cessation of work during pregnancy for $>1$ week until delivery, reduction in working hours during pregnancy, and the time in weeks to return to work after maternity leave.

Results A total of 42 women were included (21 SLE patients, 21 controls). Mean SELENA-SLEDAI before pregnancy in SLE patients was 2.6 (SD 2.3). Interruption of work for $>1$ week or completely stop working during pregnancy occurred in 10 women with SLE compared to 2 controls $(\mathrm{OR}=8.6,95 \% \mathrm{CI}[1.6-46.8], \mathrm{p}=0.012)$. From the women who completely stopped working until delivery $(n=8), 7$ women had SLE versus 1 control $(\mathrm{OR}=1.4$, 95\% CI [0.0728.1], $\mathrm{p}=0.826$ ). In addition, in women continuing work, reduction of working hours during pregnancy occurred in 5 women with SLE versus 3 controls $(\mathrm{OR}=1.9$, 95\% CI [0.49.1], $\mathrm{p}=0.436$ ).

After delivery, the median (IQR) duration of return to work after maternity leave was $4(0-6.8)$ weeks after maternity leave for women with SLE and 2 (0-4) weeks later for controls (Mann-Whitney $U$ test, $p=0.977$ ). No difference in number of women with delay of return to work after maternity leave (yes/no) was found between women with SLE and controls ( $n=9$ versus $n=11$, respectively, OR $=1.0,95 \% C I$ [0.3-3.7], $\mathrm{p}=0.973)$.
Conclusions Pregnant women with SLE more frequently completely stop working or reduce working hours compared to matched healthy controls. These findings warrant improved counseling of these women and attention of health care providers, including company doctors.

Funding Source(s): None

\section{NEUROLOGIC MANIFESTATIONS OF THE ANTIPHOSPHOLIPID SYNDROME AND RESPONSE TO HYDROXYCHLOROQUINE: A DESCRIPTIVE STUDY}

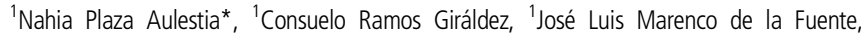
${ }^{2}$ Mercedes Romera Tellado. ${ }^{1}$ Valme Hospital (Rheumatology unit); ${ }^{2}$ Valme Hospital (Neurology unit)

\subsection{6/lupus-2019-Ism.66}

Background The primary antiphospholipid syndrome (APS) is characterized by arterial and/or venous thrombosis and pregnancy morbidity in the presence of anticardiolipin antibodies $(\mathrm{aCL})$ and/or lupus anticoagulant (LA). In addition to prothrombotic effects of aPL on the brain, there are immunologic effects with proof of direct binding of aPL to various types of brain cells, presented as cognitive dysfunction (CD), migraine, seizure, multiple sclerosis-like syndrome (MS-like), transverse myelitis (TM), movement disorders, or psychiatric symptoms.

Methods We examined 3 patients who were diagnosed primary APS.The patients were assessed with careful history taking, physical examination, blood laboratory evaluation, and MRI or head-CT.Our main objective was to describe neurologic manifestations of APS in our patients at Valme University Hospital and the response to treatment with hydroxychloroquine

Results Among the 3 patients, there was male preponderance with 3 men and no-women.The mean age of presentation was $33.66 \pm 5.2$ years (range, 2350 years) and with a current mean age of $37 \pm 14,93$ years. There was no mortality in our series. One of them debuted with sudden loss of consciousness along with jaw stiffness and posterior amnesia.The other one presented prickling sensation and involuntary movement of his right upper limb extending to right lower limbs without posterior generalization, and the third patient consulted with frontal, pulsating headache and binocular diplopia.100\% were LA positive with prolongated Activated Partial Thromboplastin Time and dilute Russell viper venom time and negative for ANA with no collagenosis sign or symptoms. Neither presented complement alteration or cerebrospinal fluid variation. We observed positivity for aCL in one patient (33\%). In 2 of 3 patients (66.66\%), cortico-subcortical space occupant lesions (SOL) were observed, some of them with contrast enhancement, mimicking demyelinating lesion, while the other patient didn't present any abnormality in the MRI images.All patients presented an appropriate response to treatment with prednisone, in downward treatment regimen, aspirin and hydroxychloroquine $200 \mathrm{mg}$ twice a day. At the four-year follow-up, all of them remain asymptomatic. We observed a lessening of SOL in MRI images due to the treatment in two patients but the third one presented new lesions due to suspension od prednisone, which was reintroduced, lessening of SOL.

Conclusions The neurological affection presented in APS can mimic multiple sclerosis symptoms and it is difficult to differentiate both entities That is why aPL determination should be part of screening tests and should not be delayed if the 